\title{
Erratum to: Fibromatosis of the breast in a 52-year-old man (2010: 10b)
}

\author{
María Yolanda Torres Sousa • María Arias Ortega • \\ María Librada Rozas Rodríguez • \\ Cristina Maria Murillo Lázaro
}

Published online: 8 February 2011

(C) European Society of Radiology 2011

\section{Erratum to: Eur Radiol}

\section{DOI 10.1007/s00330-010-1823-6}

It has now transpired that Dr Vincenzo Genchi, Bari, Italy supplied a correct answer before the closing date.

The online version of the original article can be found at http://dx.doi. org10.1007/s00330-010-1823-6.

\footnotetext{
M. Y. Torres Sousa $\cdot$ M. Arias Ortega $(\bowtie) \cdot$

M. L. Rozas Rodríguez

Servicio de Radiodiagnóstico,

Hospital General de Ciudad Real,

C/ Obispo Rafael Torija s/n, 13005 .

Ciudad Real, España

e-mail: mariaariasortega@hotmail.com

C. M. Murillo Lázaro

Servicio de Anatomía Patológica,

Hospital General de Ciudad Real,

Ciudad Real, España
} 